\title{
Symptômes associés au frottis SRAS-CoV-2-positif chez des enfants de l'Alberta
}

\author{
James A. King MSc, Tara A. Whitten PhD, Jeffrey A. Bakal PhD, Finlay A. McAlister MD MSc
}

- Citation : CMAJ 2020 January 4;193:E1-9. doi : 10.1503/cmaj.202065-f; diffusion hâtive le 24 novembre 2020

Voir la version anglaise de l'article ici : www.cmaj.ca/lookup/doi/10.1503/cmaj.202065; voir le commentaire connexe en anglais ici : www.cmaj.ca/lookup/doi/10.1503/cmaj.202568 et en français ici : www.cmaj.ca/lookup/doi/10.1503/cmaj.202568-f

\section{RÉSUMÉ}

CONTEXTE : La recherche sur les enfants atteints d'une infection à coronavirus du syndrome respiratoire aigu sévère 2 (SRAS-CoV-2) a principalement porté sur les enfants amenés aux services des urgences. Nous avons voulu identifier les symptômes plus souvent associés à un frottis SRAS-CoV-2-positif chez les enfants non hospitalisés.

MÉTHODES : Nous avons procédé à une étude observationnelle chez des enfants soumis au dépistage et suivis pour une infection à SRAS-CoV-2 confirmée sur des prélèvements de sécrétions nasales, nasopharyngées, de la gorge et autres (p. ex., aspiration nasopharyngée, sécrétions trachéales ou non spécifiées) entre le 13 avril et le 30 septembre 2020 en Alberta. Nous avons calculé les rapports de vraisemblance (RV) positifs entre les symptômes autodéclarés et les frottis
SRAS-CoV-2-positifs dans la cohorte entière et dans 3 analyses de sensibilité : tous les enfants présentant au moins 1 symptôme, tous les enfants, symptomatiques ou non, soumis au dépistage par suite d'une recherche de contacts, et tous les enfants de 5 ans et plus.

RÉSULTATS : Nous avons analysé les résultats chez 2463 enfants soumis au dépistage de l'infection à SRAS-CoV-2; 1987 enfants se sont révélés positifs et 476 négatifs. Parmi les enfants SRASCoV-2-positifs, 714 (35,9\%) n'ont déclaré aucun symptôme. Même si la toux $(24,5 \%)$ et la rhinorrhée $(19,3 \%)$ étaient les 2 symptômes les plus fréquents chez les enfants ayant contracté le SRASCoV-2, elles étaient fréquentes également chez ceux dont les résultats étaient négatifs et ne permettaient pas de prédire un résultat positif (RV positif 0,96, intervalle de confiance [IC] à $95 \% 0,81-$ 1,14 et 0,87 , IC à 95\% 0,72-1,06, respectivement). L'anosmie/agueusie (RV positif 7,33 , IC à $95 \% 3,03-17,76)$, les nausées et vomissements (RV positif 5,51, IC à 95\% 1,74-17,43), les céphalées (RV positif 2,49, IC à 95\% 1,74-3,57) et la fièvre (RV positif 1,68, IC à $95 \%$ 1,342,11 ) ont été les symptômes les plus prédictifs d'un résultat SRAS-CoV-2-positif. Le RV positif pour la combinaison anosmie et agueusie, nausées et vomissements, et céphalées était de 65,92 (IC à $95 \%$ 49,48-91,92).

INTERPRÉTATION : Environ les deux tiers des enfants déclarés SRAS-CoV-2-positifs ont manifesté des symptômes, et les symptômes les plus étroitement associés à un frottis SRAS-CoV-2-positif étaient l'anosmie/agueusie, les nausées et les vomissements, les céphalées et la fièvre.

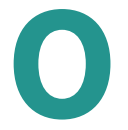

$\mathrm{n}$ entend dire que "les enfants ne sont pas de petits adultes »; cet adage s'applique sans contredit dans le contexte de la maladie à coronavirus 2019 (COVID-19). Les enfants sont moins susceptibles de contracter une infection à coronavirus du syndrome respiratoire aigu sévère (SRAS-CoV-2) que les adultes, leurs symptômes seraient moins graves et leur pronostic moins sombre ${ }^{1-4}$. Par contre, selon certaines études, cette relative bénignité de la maladie chez les enfants les rend moins susceptibles de subir un dépistage ${ }^{5}$. Trois études en 2020 ont rappelé que les symptômes de COVID-19 chez les enfants ressemblent à ceux de n'importe quelle autre affection respiratoire aiguë, soit fièvre, toux, congestion nasale, fatigue et rhinorrhée, principalement $^{6-8}$. Le 15 septembre 2020, les Centers for Disease Control and Prevention des États-Unis signalaient que la mortalité était faible chez les jeunes de moins des 21 ans (0,08\% de tous les décès) ${ }^{9}$.

En plus de connaître les symptômes qui affectent le plus souvent les enfants, les professionnels de la santé et les responsables des orientations politiques de la santé publique gagneraient à savoir lesquels peuvent être plus étroitement associés à l'infection à SRAS-CoV-2. Plusieurs régions ont mis en place des questionnaires spécifiques aux populations des milieux de garde et des écoles. Selon notre hypothèse, chez les enfants, certains symptômes pourraient être en lien plus étroit avec un résultat 
SRAS-CoV-2-positif. Nous avons donc évalué les modes de présentation des symptômes chez des enfants de l'Alberta suivis par la santé publique provinciale chez qui des prélèvements ont été effectués pour le dépistage du SRAS-CoV-2; et nous les avons comparés selon que les résultats étaient positifs ou négatifs afin d'estimer leur fréquence et leur portée pronostique dans une volumineuse cohorte et dans 3 sous-groupes.

\section{Méthodes}

\section{Population étudiée}

Nous avons conçu une étude observationnelle sur des enfants soumis au dépistage du SRAS-CoV-2 entre le 13 avril et le 30 septembre 2020 en Alberta, une province canadienne où les services de santé, tels que soins hospitaliers, consultations aux services des urgences, cliniques ambulatoires et tests de dépistage de la maladie sont financés à même les fonds publics, sans frais supplémentaires pour les usagers. Le premier cas de SRAS-CoV-2 signalé en Alberta a été découvert le 5 mars 2020; avant le 13 avril 2020, le dépistage se limitait aux voyageurs qui rentraient au pays et aux personnes hospitalisées ou soumises à un dépistage dans le cadre d'éclosions connues.

En date du 13 avril 2020, tout résident albertain symptomatique ou ayant été exposé, de l'avis de la santé publique albertaine, à un cas connu (recherche de contacts) pouvait passer un test de dépistage. Aux fins de la présente étude, nous avons inclus tous les patients pédiatriques ( $<18$ ans) SRAS-CoV-2positifs ou soumis au dépistage parce qu'ils faisaient partie d'un groupe à risque élevé (p. ex., contact étroit avec un cas connu ou contexte d'éclosion) entre le 13 avril et le 30 septembre 2020. Étant donné que certains patients ont subi plusieurs tests durant la période de l'étude, nous n'avons tenu compte que des premiers tests seulement, indépendamment du résultat, et nous avons exclu ceux qui avaient déjà subi un dépistage du SRASCoV-2 avant le 13 avril 2020. Les tests ont été effectués par RTPCR (reverse transcription-polymerase chain reaction ou test d'amplification en chaîne par polymérase couplé à une transcription inverse) sur des prélèvements de sécrétions nasales, nasopharyngées, de la gorge ou autre (p. ex., aspiration nasopharyngée, sécrétions trachéales ou non spécifiées).

\section{Sources des données}

Nous avons utilisé la base de données CDOM (Communicable Disease Outbreak Management) des Services de santé de l'Alberta pour cette étude. Les données démographiques et les symptômes ont été recueillis de manière systématique à partir des déclarations des patients ou de leurs parents ou tuteurs légaux au moyen de formulaires standardisés pour le signalement de la COVID-19 et consignés par des infirmières de la santé publique albertaine et des bénévoles étudiants en médecine. Ces renseignements ont été consignés dans la base de données CDOM. Les variables que nous avons analysées pour cette étude étaient les champs obligatoires du formulaire de signalement et, par conséquent, il ne manquait aucune donnée. Nous avons relié les données du laboratoire provincial albertain (qui enregistre les résultats des tests de SRAS-CoV-2) à celles de la base de données
CDOM à l'aide de l'identifiant permanent (IP) de chaque patient et analysé les dossiers CDOM créés dans les 5 jours de la date du prélèvement seulement. La base de données CDOM suit spécifiquement les cas de maladies transmissibles et leurs contacts, donc, les enfants amenés dans un centre de dépistage pour des symptômes, mais ayant reçu un résultat négatif n'ont pas été contactés par la santé publique albertaine pour répondre au questionnaire complet sur les symptômes.

L'entrevue standardisée portait sur la présence ou l'absence des symptômes suivants chez les patients : arthralgie et/ou myalgie, douleur thoracique, fièvre et/ou frissons (symptômes subjectifs: on ne mesurait pas la température corporelle durant la collecte des données), conjonctivite, toux, baisse de l'appétit et/ou anorexie, diarrhée, céphalées, malaises, congestion nasale, nausées et/ou vomissement, rhinorrhée, essoufflement ou gêne respiratoire, éternuements, maux de gorge ou autres. Lorsque la case " autre » était cochée, tous les symptômes déclarés par les patients ou leurs proches aidants qui ne figuraient pas dans la liste standardisée étaient notés dans une boîte de texte. Nous avons recherché dans ces boîtes de texte toute allusion à l'altération du goût (p. ex., agueusie, dysgueusie, hypogueusie, goût métallique ou modifié) ou de l'odorat (p. ex., anosmie, olfaction) et à la fatigue (p. ex., apathie, épuisement, fatigue, léthargie, ou baisse du niveau d'énergie) au moyen d'une combinaison jumelant la phonétique et un seuil établi à partir d'un algorithme de transformation en distance généralisée, auxquels avons appliqué le logiciel SAS. Le jumelage phonétique a été fait au moyen de la fonction Soundex ${ }^{10}$, l'algorithme de transformation en distance généralisée est une généralisation de la distance de Levenshtein, à partir de la fonction COMPGED du logiciel SAS. Les cas ainsi signalés ont ensuite été analysés manuellement de manière indépendante par 2 auteurs (J.A.K. et T.A.W.) qui ne connaissaient pas les résultats des frottis des patients, et tout désaccord a été résolu par l'obtention d'un consensus après discussion. Le 15 août 2020, l'anosmie/agueusie ont été ajoutées au questionnaire standardisé; aux fins de notre analyse, nous avons regroupé toute altération autodéclarée du goût ou de l'odorat à la rubrique anosmie/agueusie (le changement de fréquence des symptômes au fil du temps pour chaque symptôme est présenté à l'annexe 1 , figure $\mathrm{S} 1$, accessible en anglais au www.cmaj.ca/lookup/doi/10.1503/cmaj.202065/tab -related-content). A priori, nous avons subdivisé les groupes d'âge comme suit : $0-4,5-12$ et $13-17$ ans représentant les stades préscolaire, primaire et adolescent.

\section{Analyse statistique}

Nous avons examiné les fréquences des symptômes et leur portée pronostique chez tous les enfants suivis par la santé publique albertaine et ayant subi un dépistage du SRAS-CoV-2 par frottis, et dans 3 sous-groupes : tous les enfants ayant présenté au moins 1 symptôme et soumis au dépistage du SRAS-CoV-2, tous les enfants soumis au dépistage du SRAS-CoV-2 en raison d'un risque élevé d'exposition et tous les enfants de 5 ans ou plus soumis à un dépistage du SRAS-CoV-2 (car les enfants plus jeunes sont moins en mesure de décrire leurs symptômes). 
Nous avons calculé les rapports des cotes (RC) bruts et les rapports de vraisemblance (RV) positifs pour chaque symptôme, de même que les caractéristiques démographiques des patients, avec les intervalles de confiance IC à 95\% associés. Les rapports de vraisemblance ont été estimés, car ils étaient moins susceptibles d'être influencés par la prévalence de la maladie recherchée dans l'échantillon de population ${ }^{11}$. Nous n'avons pas calculé ces statistiques pour les prédicteurs/variables ayant une valeur prévue inférieure à 5 . Afin de mesurer l'effet de plusieurs prédicteurs ensemble sur la probabilité de résultats positifs au test, nous avons utilisé la méthode de Spiegelhalter et Knill-Jones ${ }^{12,13}$ pour calculer des RV multivariés. En bref, cela suppose de sélectionner les prédicteurs ayant un RV brut $\geq 2,0$ ou $\leq 0,5$, puis d'ajuster les logarithmes de ces RV sous forme de variables continues dans un modèle de régression logistique se présentant comme suit :

$$
\log (\text { cotes post-test })=\log (\text { cotes pré-test })+\sum_{i=1}^{n} \gamma_{i} \log \left(\mathrm{RV}_{i}\right)
$$

Les estimations des paramètres découlant de ce modèle de régression sont ensuite utilisées comme « estimations du rétrécissement » pour calculer les RV ajustés au moyen de l'équation suivante :

$$
\log \left(R V_{i} \text { ajusté }\right)=\gamma_{i} \log \left(R V_{i}\right)
$$

où $\gamma_{i}$ est l'estimation du rétrécissement obtenue par régression logistique pour le prédicteur $i$. Notez que $\gamma_{i}$ se situe généralement entre 0 et 1 , de sorte que l'effet consiste à rapprocher le $\mathrm{RV}_{i}$ ajusté plus près de 1 que le $\mathrm{RV}_{i}$ non ajusté et ce rétrécissement explique la redondance des valeurs prédictives des symptômes. Les RV multivariés globaux sont ensuite estimés sous forme de produit de ces estimations ajustées. Nous avons calculé les IC pour ces RV ajustés comme les $2,5^{\mathrm{e}}$ et $97,5^{\mathrm{e}}$ percentiles d'une distribution de rééchantillonnage (technique de bootstrap) des RV ajustés. Nous avons créé la distribution de rééchantillonnage en remettant 5000 fois notre cohorte dans l'échantillon d'origine et en remplaçant/calculant les RV ajustés (voir ci-dessus) pour chaque ensemble de données rééchantillonné. Toutes les analyses ont été effectuées avec la version SAS 9.4, la version R 3.5.2 et la trousse epiR.

\section{Approbation éthique}

Cette étude a été approuvée par le comité d'éthique de la recherche de l'Université de l'Alberta (Pro00101096) et les analyses ont porté sur des données anonymisées.

\section{Résultats}

Entre le 8 avril et le 5 octobre 2020, la base de données CDOM détenait 3474 dossiers d'enfants de moins de 18 ans en Alberta dans le cadre du suivi épidémiologique du SRAS-CoV-2 (figure 1). Après exclusion des enfants qui n'avaient pas d'identifiant permanent $(n=17)$ ou de résultats de prélèvement dans la base de données du laboratoire provincial ( $n=205), 2264$ avaient un frottis SRAS-CoV-2-positif par RT-PCR; toutefois, nous avons exclu 277 cas dont ce n'était pas le premier test ou dont le test n'avait pas été effectué dans les 5 jours de la date du signalement à la base de données CDOM; il est ainsi resté 1987 enfants SRAS-CoV-2-positifs pour l'analyse (dont 8 [0,4\%] ont été hospitalisés). Sur les 985 enfants dont le résultat était négatif, nous en avons exclu 72, car il ne s'agissait pas de leur premier test et 437 parce que le prélèvement n'avait pas été effectué dans les 5 jours de la collecte des données sur les symptômes, il restait donc 476 enfants SRAS-CoV-2-négatifs pour l'analyse (figure 1). Les tests reposaient sur des frottis analysés par RT-PCR (sécrétions nasales [2,1\%, $n=52]$, nasopharyngées [25,9\%, $n=638]$, de la gorge $[71,4 \%, n=1759]$, autres [p. ex., aspiration nasopharyngée ou sécrétions trachéales $[0,5 \%, n=12]$ ou non spécifiées $[0,1 \%, n=2])$.

Symptômes prédicteurs d'un frottis SRAS-CoV-2-positif Les symptômes les plus fréquemment observés chez les enfants SRAS-CoV-2-positifs étaient la fièvre ou les frissons (25,5\%), la toux $(24,5 \%)$ et la rhinorrhée (19,3\%) (tableau 1$)$. Sept cent quatorze enfants (35,9\%) SRAS-CoV-2-positifs se sont dits asymptomatiques. Les symptômes les plus fréquemment observés chez les enfants SRAS-CoV-2-négatifs étaient les mêmes que chez les enfants positifs : toux $(25,4 \%)$, rhinorrhée $(22,1 \%)$ fièvre ou frissons $(15,1 \%)$. Les symptômes prédicteurs de la SRAS-CoV-2-positivité dans notre analyse principale (tableau 1) étaient : anosmie/agueusie (RV positif, 7,33, IC à 95\% 3,0317,76 ), nausées/vomissements (RV positif 5,51, IC à $95 \% 1,74-$ 17,43 ), céphalées (RV positif 2,49, IC à $95 \% 1,74-3,57$ ), éternuements (RV positif 1,76, IC à $95 \%$ 1,08-2,85), fièvre/frissons (RV positif 1,68, IC à $95 \% 1,34-2,11$ ) et malaise (RV positif 1,58, IC à $95 \%$ 1,05-2,38). Aucun des autres symptômes n'a été prédicteur d'un frottis SRAS-CoV-2-positif. Le RV positif pour la combinaison anosmie/agueusie, nausées/vomissements et céphalées était de 65,92 (IC à $95 \%$ 49,48-91,92; annexe 2, tableau S1, accessible en anglais au www.cmaj.ca/lookup/doi/10.1503/ cmaj.202065/tab-related-content).

Outre les analyses liées aux symptômes, nous n'avons trouvé aucune différence liée au sexe quant à la SRAS-CoV-2-positivité ( $R V$ positif 0,93 , IC à $95 \% 0,85-1,03$ ). Une tendance générale a été observée selon laquelle les enfants plus jeunes (0-4 ans) étaient plus nombreux dans la cohorte négative (RV positif 0,77 , IC à $95 \% 0,65-0,90)$, tandis que les enfants plus âgés (13-17 ans) étaient plus nombreux dans la cohorte positive (RV positif 1,22 , IC à $95 \%$ 1,04-1,42).

\section{Analyse de sensibilité}

Nous n'avons observé aucune différence substantielle dans les estimations en restreignant l'analyse aux enfants présentant au moins 1 symptôme (tableau 2 et figure 2). Notre évaluation des enfants soumis au dépistage dans le cadre d'une recherche de contacts a généré les mêmes observations (tableau 3 et figure 2). Lorsque nous avons restreint l'analyse aux enfants de 5 ans ou plus, nous n'avons trouvé aucune différence majeure dans les estimations, même si les liens entre certains symptômes devenaient légèrement plus marqués (annexe 3, tableau S2, accessible en anglais au 


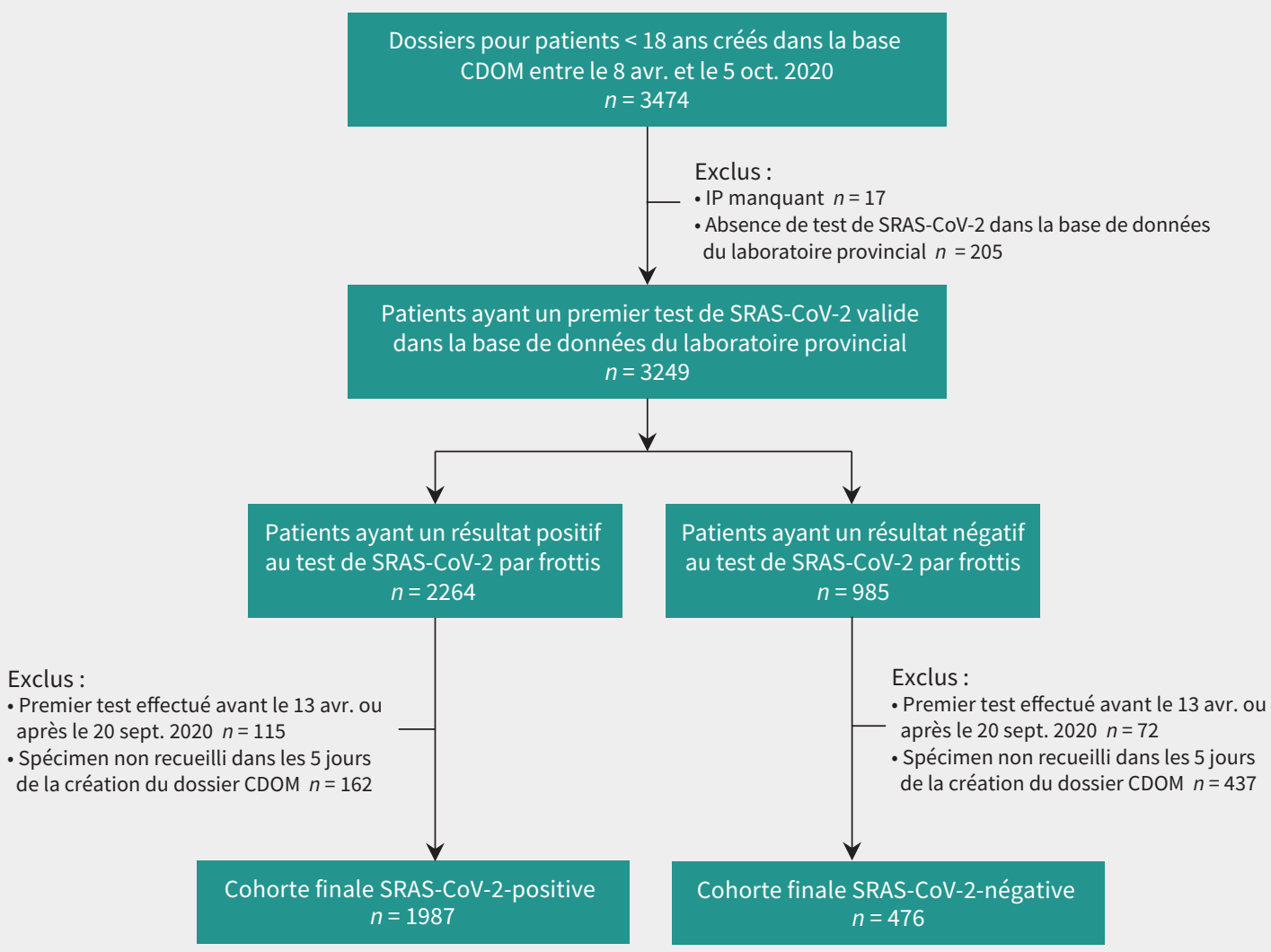

Figure 1 : Diagramme pour l'inclusion des patients. Nous avons identifié les patients de cette cohorte à l'aide de la base de données CDOM (Communicable Disease Outbreak Management) et nous les avons reliés à la base de données du laboratoire provincial de l'Alberta pour déterminer à quel moment a été effectué leur premier test de dépistage du coronavirus du syndrome respiratoire aigu sévère 2 (SRAS-CoV-2). Remarque : IP = identifiant permanent.

www.cmaj.ca/lookup/doi/10.1503/cmaj.202065/tab-related-content; figure 2). Nous avons conclu que dans toutes les analyses de sensibilité, comme dans l'analyse principale, les symptômes associés à la SRAS-CoV-2-positivité étaient l'anosmie/agueusie, les nausées/ vomissements, les céphalées et la fièvre.

\section{Interprétation}

Dans une cohorte d'enfants soumis au dépistage du SRAS-CoV-2, nous avons constaté que les symptômes étroitement associés à un frottis SRAS-CoV-2-positif étaient l'anosmie/agueusie, les nausées/vomissements, les céphalées et la fièvre, tandis que la toux, les maux de gorge, la rhinorrhée, la congestion nasale ou la diarrhée n'y étaient pas associés. Les responsables de l'administration des questionnaires de dépistage pour les écoles ou les garderies pourraient souhaiter envisager une réévaluation des symptômes qu'ils recherchent et n'inclure que ceux qui sont plus étroitement associés à la SRAS-CoV-2-positivité. Les RV positifs pour les 4 symptômes les plus étroitement associés à l'infection ont été similaires dans toutes nos analyses de sensibilité et sont donc pertinents si l'on procède à un dépistage chez des enfants symptomatiques (tableau 2) ou si l'on interroge ceux qui ont subi le test dans le contexte d'une recherche de contacts (tableau 3).

Comme lors d'autres études ${ }^{6,7,14}$, la toux, la fièvre et la rhinorrhée ont été les symptômes les plus fréquents dans notre cohorte; seule la fièvre a été associée à des résultats positifs au test de dépistage du SRAS-CoV-2. Par contre, dans notre étude, la fréquence de ces symptômes (19-26\%) était inférieure à celle rapportée par les études effectuées en milieu hospitalier, alors que la toux était présente chez $39 \%{ }^{4}$ et $49 \%{ }^{15}$ des patients, et la fièvre chez $42 \%{ }^{15}, 70 \%^{4}$ et $74 \%^{16}$. Notre cohorte extrahospitalière inclut probablement des cas plus légers que les cohortes hospitalières, ce qui explique ces fréquences moindres. En revanche, la rhinorrhée était plus fréquente dans notre cohorte $(19 \%)$ que dans une cohorte hospitalière $(8 \%)^{15}$, même si cela n'a pas été constant d'une étude à l'autre ${ }^{16}$. Notre fréquence des symptômes est également moindre que celle de rapports récents de l'Agence de la santé publique du Canada (ASPC) en ce qui concerne les symptômes chez les enfants canadiens atteints de COVID-19 (où la toux a été signalée chez $49 \%$, la fièvre chez $46 \%$ et la rhinorrhée chez $40 \%$ ), mais ici, les données de l'ASPC ne faisaient état des symptômes que pour $26 \%$ des cas, avec une grande variation entre les régions ${ }^{14}$. 
Tableau 1 : Caractéristiques et symptômes de référence chez des enfants soumis au dépistage de l'infection à coronavirus du syndrome respiratoire aigu sévère 2

\begin{tabular}{|c|c|c|c|c|}
\hline Variables & $\begin{array}{c}\mathrm{N}^{\text {bre }}(\%) \text { chez les } \\
\text { patients SRAS-CoV-2- } \\
\text { positifs* } \\
n=1987\end{array}$ & $\begin{array}{c}\mathrm{N}^{\text {bre }}(\%) \text { chez les } \\
\text { patients SRAS-CoV-2- } \\
\text { négatifs* } \\
n=476\end{array}$ & $\begin{array}{l}\text { RC non ajusté } \\
\text { (IC à } 95 \%)\end{array}$ & $\begin{array}{l}\text { RV positif non ajust } \\
\text { pour l'infection à } \\
\text { SRAS-CoV-2 } \\
\text { (IC à } 95 \% \text { ) }\end{array}$ \\
\hline \multicolumn{5}{|l|}{ Caractéristiques démographiques } \\
\hline Âge moyen \pm É.-T., ans & $9,3 \pm 5,2$ & $8,5 \pm 5,3$ & S.O. & S.O. \\
\hline Âge, $0-4$ ans & $458(23,0)$ & $143(30,0)$ & $0,70(0,56-0,87)$ & $0,77(0,65-0,90)$ \\
\hline Âge, 5-12 ans & $849(42,7)$ & $199(41,8)$ & $1,04(0,85-1,27)$ & $1,02(0,91-1,15)$ \\
\hline Âge, $13-17$ ans & $680(34,2)$ & $134(28,2)$ & $1,33(1,07-1,65)$ & $1,22(1,04-1,42)$ \\
\hline Sexe masculin & $989(49,8)$ & $254(53,4)$ & $0,87(0,71-1,06)$ & $0,93(0,85-1,03)$ \\
\hline \multicolumn{5}{|l|}{ Symptômes } \\
\hline Anosmie/agueusie & $153(7,7)$ & $5(1,1)$ & $7,86(3,21-19,26)$ & $7,33(3,03-17,76)$ \\
\hline Nausées/vomissements & $69(3,5)$ & Ignoré, $n<5$ & $5,67(1,78-18,10)$ & $5,51(1,74-17,43)$ \\
\hline Céphalées & $312(15,7)$ & $30(6,3)$ & $2,77(1,88-4,09)$ & $2,49(1,74-3,57)$ \\
\hline Baisse de l'appétit/anorexie & $75(3,8)$ & $10(2,1)$ & $1,83(0,94-3,56)$ & $1,80(0,94-3,45)$ \\
\hline Éternuements & $132(6,6)$ & $18(3,8)$ & $1,81(1,09-2,99)$ & $1,76(1,08-2,85)$ \\
\hline Fièvre ou frissons & $506(25,5)$ & $72(15,1)$ & $1,92(1,46-2,51)$ & $1,68(1,34-2,11)$ \\
\hline $\begin{array}{l}\text { Douleurs musculaires/ } \\
\text { articulaires (myalgie, arthralgie) }\end{array}$ & $56(2,8)$ & $8(1,7)$ & $1,70(0,80-3,58)$ & $1,68(0,80-3,49)$ \\
\hline Malaise & $165(8,3)$ & $25(5,3)$ & $1,63(1,06-2,52)$ & $1,58(1,05-2,38)$ \\
\hline Congestion nasale & $241(12,1)$ & $46(9,7)$ & $1,29(0,93-1,80)$ & $1,26(0,93-1,69)$ \\
\hline Fatigue & $31(1,6)$ & $6(1,3)$ & $1,24(0,51-2,99)$ & $1,24(0,52-2,95)$ \\
\hline Gêne respiratoire/dyspnée & $31(1,6)$ & $6(1,3)$ & $1,24(0,51-2,99)$ & $1,24(0,52-2,95)$ \\
\hline Maux de gorge & $311(15,7)$ & $67(14,1)$ & $1,13(0,85-1,51)$ & $1,11(0,87-1,42)$ \\
\hline Diarrhée & $57(2,9)$ & $13(2,7)$ & $1,05(0,57-1,94)$ & $1,05(0,58-1,90)$ \\
\hline Toux & $486(24,5)$ & $121(25,4)$ & $0,95(0,75-1,20)$ & $0,96(0,81-1,14)$ \\
\hline Rhinorrhée & $383(19,3)$ & $105(22,1)$ & $0,84(0,66-1,08)$ & $0,87(0,72-1,06)$ \\
\hline Douleur thoracique & $15(0,8)$ & Ignoré, $n<5$ & S.O. & S.o. \\
\hline Conjonctivite & $11(0,6)$ & Ignoré, $n<5$ & S.O. & S.O. \\
\hline Asymptomatique & $714(35,9)$ & $211(44,3)$ & $0,70(0,58-0,86)$ & $0,81(0,72-0,91)$ \\
\hline
\end{tabular}

Remarque : É.-T. = écart-type, IC = intervalle de confiance, RC = rapport des cotes, RV = rapport de vraisemblance, $\mathrm{S} . \mathrm{O}$. = sans objet, $\mathrm{SRAS}-\mathrm{CoV}-2=$ coronavirus du syndrome respiratoire aigu sévère 2 .

*À moins d'indication contraire.

Nous avons aussi constaté que $36 \%$ des enfants SRAS-CoV2-positifs ne déclaraient aucun symptôme, ce qui s'apparente aux $27,2 \%$ des enfants SRAS-CoV-2-positifs asymptomatiques des plus récentes données de l'ASPC et aux $27 \%$ rapportés dans une analyse prépubliée concernant des enfants d'âge scolaire en Suisse $^{14,17}$. Il importe de noter qu'il s'agit probablement d'une sous-estimation de la prévalence réelle de l'infection à SRASCoV-2 asymptomatique, étant donné que les patients asymptomatiques étaient beaucoup moins susceptibles d'être soumis au test que les patients symptomatiques. Nos observations selon lesquelles plusieurs symptômes d'infection virale ont la même prévalence chez les enfants, que les résultats soient négatifs ou positifs, concordent aussi avec les conclusions de l'analyse prépubliée sur la cohorte suisse ${ }^{17}$.
Même si le tableau clinique varie selon le groupe d'âge (adultes c. enfants), plusieurs des symptômes que nous avons jugés en lien étroit avec un frottis SRAS-CoV-2-positif par RT-PCR chez les enfants sont semblables à ceux d'une population comparable d'adultes vus en consultation externe ${ }^{18}$. Après révision, l'anosmie/agueusie ont été les prédicteurs les plus fiables, tant chez les adultes que les enfants, même si leur fréquence et l'ampleur du RV positif étaient moindres chez ces derniers. Par contre, les céphalées s'accompagnaient d'un RV positif plus élevé quand les frottis SRAS-CoV-2 étaient positifs chez les enfants, ce qu'il importe de savoir lors du dépistage d'une possible infection chez cette population. Il faut rappeler que la plupart des symptômes associés à la SRAS-CoV-2-positivité sont peu fréquents chez les enfants et auraient donc une piètre sensibilité 
Tableau 2 : Caractéristiques et symptômes de référence chez des enfants de l'Alberta présentant au moins 1 symptôme et soumis au dépistage de l'infection à coronavirus du syndrome respiratoire aigu sévère 2

\begin{tabular}{|c|c|c|c|c|}
\hline Variables & $\begin{array}{c}N^{\text {bre }}(\%) \text { chez les } \\
\text { patients SRAS-CoV-2- } \\
\text { positifs } \\
n=1273\end{array}$ & $\begin{array}{c}\mathrm{N}^{\text {bre }}(\%) \text { chez les } \\
\text { patients SRAS-CoV-2- } \\
\text { négatifs* } \\
n=265\end{array}$ & $\begin{array}{l}\text { RC non ajusté } \\
\text { (IC à } 95 \%)\end{array}$ & $\begin{array}{l}\text { RV positif non ajuste } \\
\text { pour l'infection à } \\
\text { SRAS-CoV-2 } \\
\text { (IC à } 95 \% \text { ) }\end{array}$ \\
\hline \multicolumn{5}{|l|}{ Caractéristiques démographiques } \\
\hline Âge moyen \pm É.-T., ans & $9,8 \pm 5,4$ & $8,4 \pm 5,3$ & S.O. & S.O. \\
\hline Âge, $0-4$ ans & $281(22,1)$ & $80(30,2)$ & $0,66(0,49-0,88)$ & $0,73(0,59-0,90)$ \\
\hline Âge, 5-12 ans & $482(37,9)$ & $110(41,5)$ & $0,86(0,66-1,12)$ & $0,91(0,78-1,07)$ \\
\hline Âge, $13-17$ ans & $510(40,1)$ & $75(28,3)$ & $1,69(1,27-2,26)$ & $1,42(1,16-1,73)$ \\
\hline Sexe masculin & $629(49,4)$ & $142(53,6)$ & $0,85(0,65-1,10)$ & $0,92(0,81-1,04)$ \\
\hline \multicolumn{5}{|l|}{ Symptômes } \\
\hline Anosmie/agueusie & $153(12,0)$ & $5(1,9)$ & $7,10(2,89-17,49)$ & $6,37(2,64-15,37)$ \\
\hline Nausées/vomissements & $69(5,4)$ & Ignoré, $n<5$ & $5,00(1,56-16,02)$ & $4,79(1,52-15,10)$ \\
\hline Céphalées & $312(24,5)$ & $30(11,3)$ & $2,54(1,70-3,80)$ & $2,16(1,52-3,07)$ \\
\hline Baisse de l'appétit/anorexie & $71(5,6)$ & $9(3,4)$ & $1,68(0,83-3,41)$ & $1,64(0,83-3,24)$ \\
\hline Éternuements & $132(10,4)$ & $18(6,8)$ & $1,59(0,95-2,65)$ & $1,53(0,95-2,45)$ \\
\hline Fièvre ou frissons & $506(39,7)$ & $72(27,2)$ & $1,77(1,32-2,37)$ & $1,46(1,19-1,80)$ \\
\hline $\begin{array}{l}\text { Douleurs musculaires/ } \\
\text { articulaires (myalgie, arthralgie) }\end{array}$ & $56(4,4)$ & $8(3,0)$ & $1,48(0,70-3,14)$ & $1,46(0,70-3,02)$ \\
\hline Malaise & $165(13,0)$ & $25(9,4)$ & $1,43(0,92-2,23)$ & $1,37(0,92-2,05)$ \\
\hline Congestion nasale & $241(18,9)$ & $46(17,4)$ & $1,11(0,79-1,57)$ & $1,09(0,82-1,45)$ \\
\hline Fatigue & $31(2,4)$ & $6(2,3)$ & $1,08(0,44-2,61)$ & $1,08(0,45-2,55)$ \\
\hline Gêne respiratoire/dyspnée & $31(2,4)$ & $6(2,3)$ & $1,08(0,44-2,61)$ & $1,08(0,45-2,55)$ \\
\hline Maux de gorge & $311(24,4)$ & $67(25,3)$ & $0,96(0,70-1,30)$ & $0,97(0,77-1,21)$ \\
\hline Diarrhée & $57(4,5)$ & $13(4,9)$ & $0,91(0,49-1,68)$ & $0,91(0,51-1,64)$ \\
\hline Toux & $486(38,2)$ & $121(45,7)$ & $0,73(0,56-0,96)$ & $0,84(0,72-0,97)$ \\
\hline Rhinorrhée & $383(30,1)$ & $105(39,6)$ & $0,66(0,50-0,86)$ & $0,76(0,64-0,90)$ \\
\hline Douleur thoracique & $15(1,2)$ & Ignoré, $n<5$ & S.O. & S.O. \\
\hline Conjonctivite & $11(0,9)$ & Ignoré, $n<5$ & S.O. & S.O. \\
\hline
\end{tabular}

Remarque : É.-T. = écart-type, IC = intervalle de confiance, $\mathrm{RC}=$ rapport des cotes, $\mathrm{RV}=$ rapport de vraisemblance, $\mathrm{S} . \mathrm{O}$. = sans objet, $\mathrm{SRAS}-\mathrm{CoV}-2=$ coronavirus du syndrome respiratoire aigu sévère 2 .

*À moins d'indication contraire.

pour un éventuel test de dépistage. Par exemple, parmi les symptômes prédicteurs d'un prélèvement SRAS-CoV-2-positif, la fièvre a été le plus souvent observée chez des patients positifs, et n'affectait que $26 \%$ des enfants.

Un des atouts de notre étude est sa cohorte de patients extrahospitalière, qui a donné un tableau clinique plus complet des enfants selon diverses intensités de la maladie plutôt que de se limiter aux enfants hospitalisés ou ayant consulté aux services des urgences. Il importe également de prévenir la transmission du SRAS-CoV-2 dans les milieux de garde et les écoles pour protéger la santé et la sécurité des enfants et réduire la propagation de la maladie à la communauté élargie. Étant donné que certaines mesures d'atténuation des risques pour la santé (p. ex., le port du couvre-visage et la distanciation physique) posent des défis particuliers chez les jeunes enfants, la surveil- lance des symptômes évocateurs d'une infection à SRAS-CoV-2 avant leur entrée à l'école ou à la garderie pourrait contribuer à réduire les risques de transmission de la maladie. Même si selon nos résultats, certains symptômes peuvent être utiles pour identifier de possibles infections à SRAS-CoV-2, de futures études pourraient générer des renseignements utiles si elles utilisaient des protocoles basés dans la population ou si elles portaient sur un échantillon représentatif d'enfants SRAS-CoV-2-négatifs (non seulement ceux identifiés lors de recherches de contacts).

\section{Limites de l'étude}

Notre étude comportait certaines limites notables. Nous n'avions pas de données sur les symptômes de la plupart des enfants dont les prélèvements étaient négatifs par RT-PCR en 


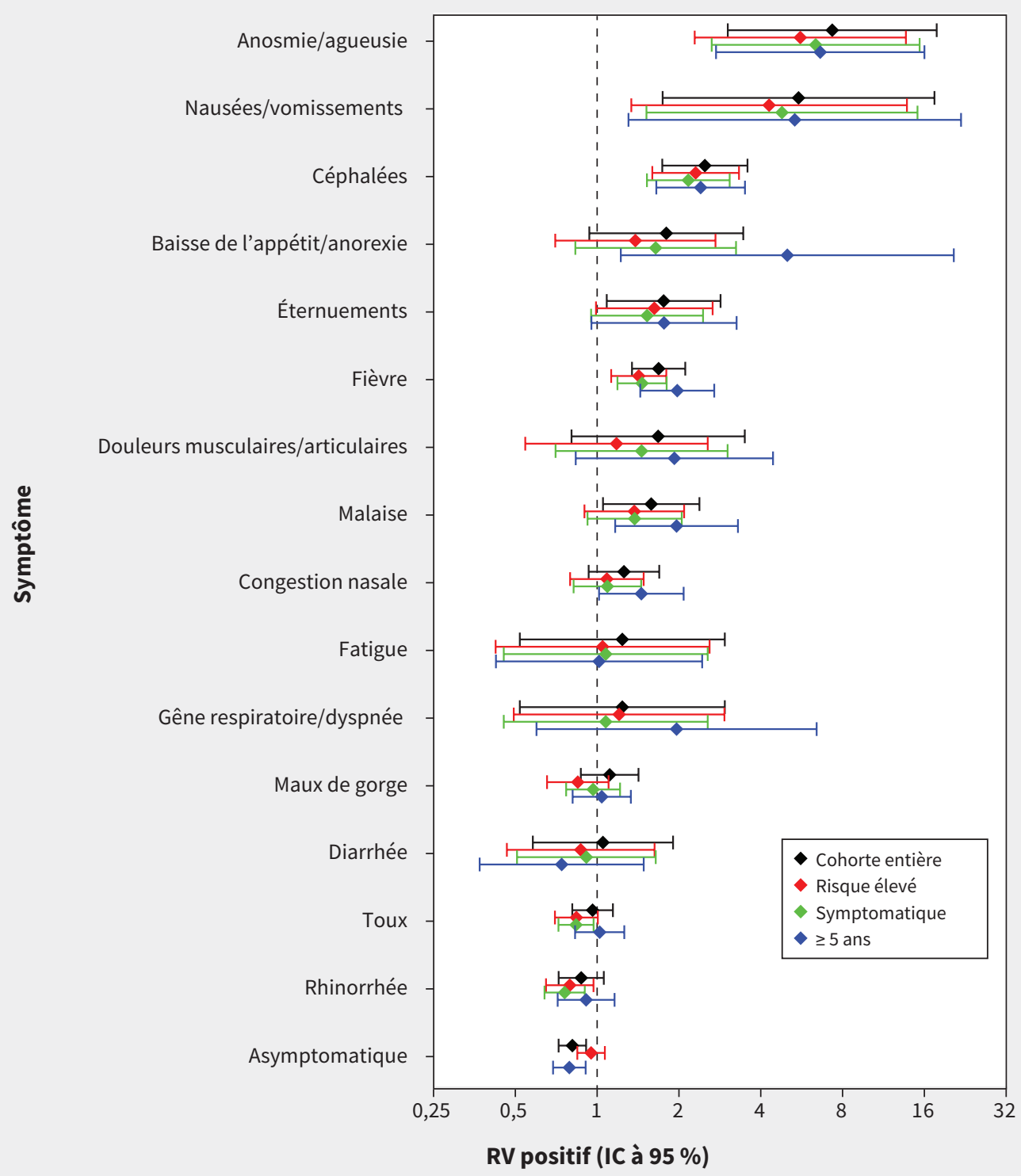

Figure 2 : Rapports de vraisemblance (RV) positifs pour les symptômes associés aux frottis coronavirus du syndrome respiratoire aigu sévère 2 (SRASCoV-2)-positifs chez des enfants de l'Alberta. Les estimations des RV positifs et les intervalles de confiance (IC) à $95 \%$ associés sont présentés pour la cohorte entière (en noir), pour certains enfants jugés à haut risque d'exposition par suite d'une recherche de contacts (en rouge), pour ceux qui présentaient au moins 1 symptôme (en vert) et ceux qui avaient 5 ans ou plus (en bleu).

Alberta. La santé publique provinciale utilise la base de données CDOM pour suivre spécifiquement les cas de maladies transmissibles et leurs contacts. Ainsi, les personnes s'étant présentées dans un centre de dépistage pour des symptômes et ayant obtenu un résultat négatif n'ont pas été contactées par la santé publique albertaine pour répondre au questionnaire sur les symptômes. Dans notre échantillon, les enfants à haut risque d'exposition qui étaient SRAS-CoV-2-négatifs ont donc été identifiés dans le contexte d'une recherche de contacts. L'inclusion de tous les cas positifs et d'un groupe limité de cas négatifs seulement est une source de biais potentiel dans notre population. Nous avons voulu atténuer ce biais en mettant l'accent sur les RV positifs plutôt que sur les RC ou les valeurs prédictives positives et en procédant à une analyse de sensibilité qui restreignait la cohorte positive aux patients jugés à risque élevé à la suite d'une recherche de contacts, et les résultats ont été similaires (figure 2); mais il faut garder cette limite à l'esprit lorsqu'on envisage de généraliser nos résultats à tous les enfants testés pour l'infection à SRASCoV-2. Il nous manquait aussi des données sur les symptômes 
Tableau 3 : Caractéristiques et symptômes de référence chez des enfants de l'Alberta soumis au dépistage de l'infection à coronavirus du syndrome respiratoire aigu sévère 2 en raison d'un risque élevé d'exposition

\begin{tabular}{|c|c|c|c|c|}
\hline Variables & $\begin{array}{c}\mathrm{N}^{\text {bre }}(\%) \text { chez les } \\
\text { patients SRAS-CoV-2- } \\
\text { positifs* } \\
n=1515\end{array}$ & $\begin{array}{c}\mathrm{N}^{\text {bre }}(\%) \text { chez les } \\
\text { patients SRAS-CoV-2- } \\
\text { négatifs* } \\
n=476\end{array}$ & $\begin{array}{l}\text { RC non ajusté } \\
\text { (IC à } 95 \%)\end{array}$ & $\begin{array}{l}\text { RV positif non ajusté } \\
\text { pour l'infection à } \\
\text { SRAS-CoV-2 } \\
\text { (IC à } 95 \% \text { ) }\end{array}$ \\
\hline \multicolumn{5}{|l|}{ Caractéristiques démographiques } \\
\hline Âge moyen \pm É.-T., ans & $9,1 \pm 5,2$ & $8,5 \pm 5,3$ & S.O. & S.O. \\
\hline Âge, $0-4$ ans & $361(23,8)$ & $143(30,0)$ & $0,73(0,58-0,92)$ & $0,79(0,67-0,93)$ \\
\hline Âge, 5-12 ans & $672(44,4)$ & $199(41,8)$ & $1,11(0,90-1,37)$ & $1,06(0,94-1,20)$ \\
\hline Âge, $13-17$ ans & $482(31,8)$ & $134(28,2)$ & $1,19(0,95-1,49)$ & $1,13(0,96-1,33)$ \\
\hline Sexe, masculin & $755(49,8)$ & $254(53,4)$ & $0,87(0,71-1,07)$ & $0,93(0,85-1,03)$ \\
\hline \multicolumn{5}{|l|}{ Symptômes } \\
\hline Anosmie/agueusie & $89(5,9)$ & $5(1,1)$ & $5,88(2,37-14,56)$ & $5,59(2,29-13,69)$ \\
\hline Nausées/vomissements & $41(2,7)$ & Ignoré, $n<5$ & $4,39(1,35-14,23)$ & $4,29(1,34-13,80)$ \\
\hline Céphalées & $220(14,5)$ & $30(6,3)$ & $2,53(1,70-3,75)$ & $2,30(1,60-3,33)$ \\
\hline Baisse de l'appétit/anorexie & $44(2,9)$ & $10(2,1)$ & $1,39(0,70-2,79)$ & $1,38(0,70-2,73)$ \\
\hline Éternuements & $93(6,1)$ & $18(3,8)$ & $1,66(0,99-2,79)$ & $1,62(0,99-2,66)$ \\
\hline Fièvre ou frissons & $326(21,5)$ & $72(15,1)$ & $1,54(1,16-2,03)$ & $1,42(1,13-1,80)$ \\
\hline $\begin{array}{l}\text { Douleurs musculaires/ } \\
\text { articulaires (myalgie, arthralgie) }\end{array}$ & $30(2,0)$ & $8(1,7)$ & $1,18(0,54-2,60)$ & $1,18(0,54-2,55)$ \\
\hline Malaises & $109(7,2)$ & $25(5,3)$ & $1,40(0,89-2,19)$ & $1,37(0,90-2,09)$ \\
\hline Congestion nasale & $159(10,5)$ & $46(9,7)$ & $1,10(0,78-1,55)$ & $1,09(0,80-1,48)$ \\
\hline Fatigue & $20(1,3)$ & $6(1,3)$ & $1,05(0,42-2,62)$ & $1,05(0,42-2,59)$ \\
\hline Gêne respiratoire/dyspnée & $23(1,5)$ & $6(1,3)$ & $1,21(0,49-2,98)$ & $1,20(0,49-2,94)$ \\
\hline Maux de gorge & $181(11,9)$ & $67(14,1)$ & $0,83(0,61-1,12)$ & $0,85(0,65-1,10)$ \\
\hline Diarrhée & $36(2,4)$ & $13(2,7)$ & $0,87(0,46-1,65)$ & $0,87(0,47-1,63)$ \\
\hline Toux & $323(21,3)$ & $121(25,4)$ & $0,80(0,63-1,01)$ & $0,84(0,70-1,01)$ \\
\hline Rhinorrhée & $265(17,5)$ & $105(22,1)$ & $0,75(0,58-0,97)$ & $0,79(0,65-0,97)$ \\
\hline Douleur thoracique & $9(0,6)$ & Ignoré, $n<5$ & S.O. & S.O. \\
\hline Conjonctivite & $8(0,5)$ & Ignoré, $n<5$ & S.O. & S.O. \\
\hline Asymptomatique & $638(42,1)$ & $211(44,3)$ & $0,91(0,74-1,12)$ & $0,95(0,85-1,07)$ \\
\hline
\end{tabular}

Remarque : É.-T. = écart-type, IC = intervalle de confiance, RC = rapport des cotes, RV = rapport de vraisemblance, $\mathrm{S} . \mathrm{O}$. = sans objet, $\mathrm{SRAS}-\mathrm{CoV}-2=$ coronavirus du syndrome respiratoire aigu sévère 2 .

*À moins d'indication contraire.

des enfants ayant contracté le SRAS-CoV-2 sans subir de dépistage. Cela pourrait expliquer pourquoi les enfants de moins de 5 ans étaient moins susceptibles d'avoir des résultats positifs puisqu'ils sont peut-être simplement passés à côté du dépistage si leurs symptômes ne s'accompagnaient pas d'un risque perçu. Durant la période de notre étude, les critères de dépistage ont évolué en Alberta en fonction de la réponse de la santé publique provinciale; cela peut avoir joué sur le nombre d'enfants asymptomatiques soumis au dépistage. En outre, les symptômes étaient autodéclarés, ce qui comporte un risque de sous-signalement des symptômes moins connus, et les enfants plus jeunes pourraient avoir été moins aptes à décrire clairement leurs symptômes. La plupart des questionnaires sur les symptômes ont été remplis au moment du contact initial avec l'équipe de la santé publique albertaine; même si les dossiers CDOM pouvaient être mis à jour par la suite, nous ne pouvons pas vérifier à quelle fréquence cela s'est produit. Nous ne disposions pas de renseignements sur les diagnostics d'allergies saisonnières ou d'asthme chez les enfants soumis aux tests, ce qui peut avoir mené à une fausse attribution des symptômes. Finalement, il faut reconnaître que, comme dans toute étude, certains liens réels peuvent être passés inaperçus en raison d'une puissance statistique insuffisante.

\section{Conclusion}

Étant donné que le tiers des patients pédiatriques qui obtiennent des résultats positifs au dépistage du SRAS-CoV-2 ne manifestent aucun symptôme, il peut être difficile de cibler ceux qui risquent 
d'être infectés. Notre étude énumère certains symptômes qui peuvent être utiles comme prédicteurs des infections à SRASCoV-2 chez les enfants. Plus spécifiquement, l'altération des sens de l'odorat ou du goût, les nausées/les vomissements, les céphalées et la fièvre ont été les seuls symptômes étroitement associés à un résultat positif dans toutes nos analyses. Plusieurs autres symptômes pseudogrippaux (comme la toux, la rhinorrhée et les maux de gorge) étaient aussi fréquents sinon plus fréquents chez les enfants SRAS-CoV-2-négatifs. Cette information pourrait être utile à ceux qui élaborent des questionnaires de dépistage pour les écoles et les garderies.

\section{Références}

1. Mehta NS, Mytton OT, Mullins EWS, et al. SARS-CoV-2 (COVID-19): What do we know about children? A systematic review. Clin Infect Dis 2020 May 11 [Cyberpublication avant impression]. doi : 10.1093/cid/ciaa556.

2. Li Q, Guan X, Wu P, et al. Early transmission dynamics in Wuhan, China, of novel coronavirus-infected pneumonia. N Engl J Med 2020;382:1199-207.

3. Wu Z, McGoogan JM. Characteristics of and important lessons from the coronavirus disease 2019 (COVID-19) outbreak in China: summary of a report of 72314 cases from the Chinese Center for Disease Control and Prevention. JAMA 2020;323:1239-42.

4. Swann OV, Holden KA, Turtle L, et al. Clinical characteristics of children and young people admitted to hospital with COVID-19 in United Kingdom: prospective multicentre observational cohort study. BMJ 2020;370:m3249.

5. Bi Q, Wu Y, Mei S, et al. Epidemiology and transmission of COVID-19 in 391 cases and 1286 of their close contacts in Shenzhen, China: a retrospective cohort study. Lancet Infect Dis 2020;20:911-9.

6. Ludvigsson JF. Systematic review of COVID-19 in children shows milder cases and a better prognosis than adults. Acta Paediatr 2020;109:1088-95.
7. Chen Z-M, Fu J-F, Shu Q, et al. Diagnosis and treatment recommendations for pediatric respiratory infection caused by the 2019 novel coronavirus. World $J$ Pediatr 2020;16:240-6.

8. Yang Z-D, Zhou G-J, Jin R-M, et al. Clinical and transmission dynamics characteristics of 406 children with coronavirus disease 2019 in China: a review. J Infect 2020;81:e11-5.

9. Bixler D, Miller AD, Mattison CP, et al.; Pediatric Mortality Investigation Team. SARS-CoV-2-associated deaths among persons aged < 21 years: United States, February 12-July 31, 2020. MMWR Morb Mortal Wkly Rep 2020;69:1324-9.

10. Knuth DE. The art of computer programming: Vol. 3 - Sorting and searching. 2nd ed. Boston: Addison-Wesley Professional; 1998.

11. McGee S. Simplifying likelihood ratios. J Gen Intern Med 2002;17:646-9.

12. Spiegelhalter DJ, Knill-Jones RP. Statistical and knowledge-based approaches to clinical decision-support systems, with an application in gastroenterology. J R Stat Soc [Ser A] 1984;147:35-58.

13. Chan SF, Deeks JJ, Macaskill P, et al. Three methods to construct predictive models using logistic regression and likelihood ratios to facilitate adjustment for pretest probability give similar results. J Clin Epidemiol 2008;61:52-63.

14. Table 13-10-0781-01: Detailed preliminary information on confirmed cases of COVID-19 (Revised), Public Health Agency of Canada. Ottawa: Statistics Canada; 2020. Accessible ici : www150.statcan.gc.ca/t1/tbl1/en/tv.action?pid=1310078101 (consulté le 13 oct. 2020).

15. Lu X, Zhang L, Du H, et al.; Chinese Pediatric Novel Coronavirus Study Team. SARS-CoV-2 infection in children. N Engl J Med 2020;382:1663-5.

16. Chan JF-W, Yuan S, Kok K-H, et al. A familial cluster of pneumonia associated with the 2019 novel coronavirus indicating person-to-person transmission: a study of a family cluster. Lancet 2020;395:514-23.

17. Ulyte A, Radtke T, Abela IA, et al. Variation in SARS-CoV-2 seroprevalence in school-children across districts, schools and classes [pré-impression]. medRxiv 2020 Sept. 18. doi : 10.1101/2020.09.18.20191254.

18. McAlister FA, Whitten TA, King JA, et al. Which adults presenting for SARSCoV-2 testing are most likely to have a positive swab? A population-based cohort of 15,132 adults from Alberta, Canada. Chest. In press.
Intérêts concurrents : Aucun intérêt concurrent n'a été déclaré.

Cet article a été révisé par des pairs.

Affiliations : Alberta Strategy for Patient Oriented Research Support Unit Data Platform et Provincial Research Data Services (King, Whitten), Services de santé de l'Alberta, Calgary, Alb.; Alberta Strategy for Patient Oriented Research Support Unit Data Platform (Bakal, McAlister) et Provincial Research Data Services (Bakal), Services de santé de l'Alberta, Edmonton, Alb.; Division de médecine interne générale (McAlister), Université de l'Alberta, Edmonton, Alb.

Collaborateurs : Tous les auteurs ont collaboré à l'élaboration et à la conception de l'étude. James King et Tara Whitten ont procédé à l'analyse et rédigé l'ébauche du manuscrit. Jeffrey Bakal et Finlay McAlister ont révisé le manuscrit de manière critique pour le contenu intellectuel important. Tous les auteurs ont donné leur approbation finale pour la version soumise pour publication et assument l'entière responsabilité de tous les aspects du travail. James King et Tara Whitten ont contribué également à titre de coauteurs principaux.

Financement : Finlay McAlister est détenteur de la Chaire de recherche en médecine cardiovasculaire des Services de santé de l'Alberta et ce projet a bénéficié de l'appui de l'unité de soutien de l'Alberta Strategy for Patient Oriented Research.

Partage des données : L'ensemble de données tirées de cette étude est conservé de manière sécuritaire sous forme encodée auprès des Services de santé de l'Alberta. Des ententes sur le partage des données empêchent les Services de santé de l'Alberta de rendre les données accessibles au public, mais l'accès peut être accordé aux personnes répondant aux critères d'accès confidentiels préétablis. Veuillez communiquer avec SPOR.DATA@ahs.ca pour plus de renseignements.

Propriété intellectuelle du contenu : Il s'agit d'un article en libre accès distribué conformément aux modalités de la licence Creative Commons Attribution (CC BY-NC-ND 4.0), qui permet l'utilisation, la diffusion et la reproduction dans tout médium à la condition que la publication originale soit adéquatement citée, que l'utilisation se fasse à des fins non commerciales (c.-à-d., recherche ou éducation) et qu'aucune modification ni adaptation n'y soit apportée. Voir : https://creativecommons. org/licenses/by-nc-nd/4.0/

Remerciements : Nous remercions Tanya Masson-McCrory, éducatrice clinique au programme CDOM (Communicable Disease Outbreak Management) des Services de santé de l'Alberta et les bénévoles étudiants en médecine qui ont aidé à la collecte des données sur les symptômes des patients conservées par le programme CDOM.

Avis : Cette étude se fonde en partie sur des données fournies par le ministère de la Santé de l'Alberta et par les Services de santé de l'Alberta. L'interprétation et les conclusions présentées ici sont celles des chercheurs et ne représentent pas les vues du gouvernement de l'Alberta ni des Services de santé de l'Alberta. Ni le gouvernement de l'Alberta ni Les Services de santé de l'Alberta n'expriment d'opinion sur cette étude.

Accepté : Le 9 novembre 2020

Correspondance : Finlay McAlister, finlay.mcalister@ualberta.ca 